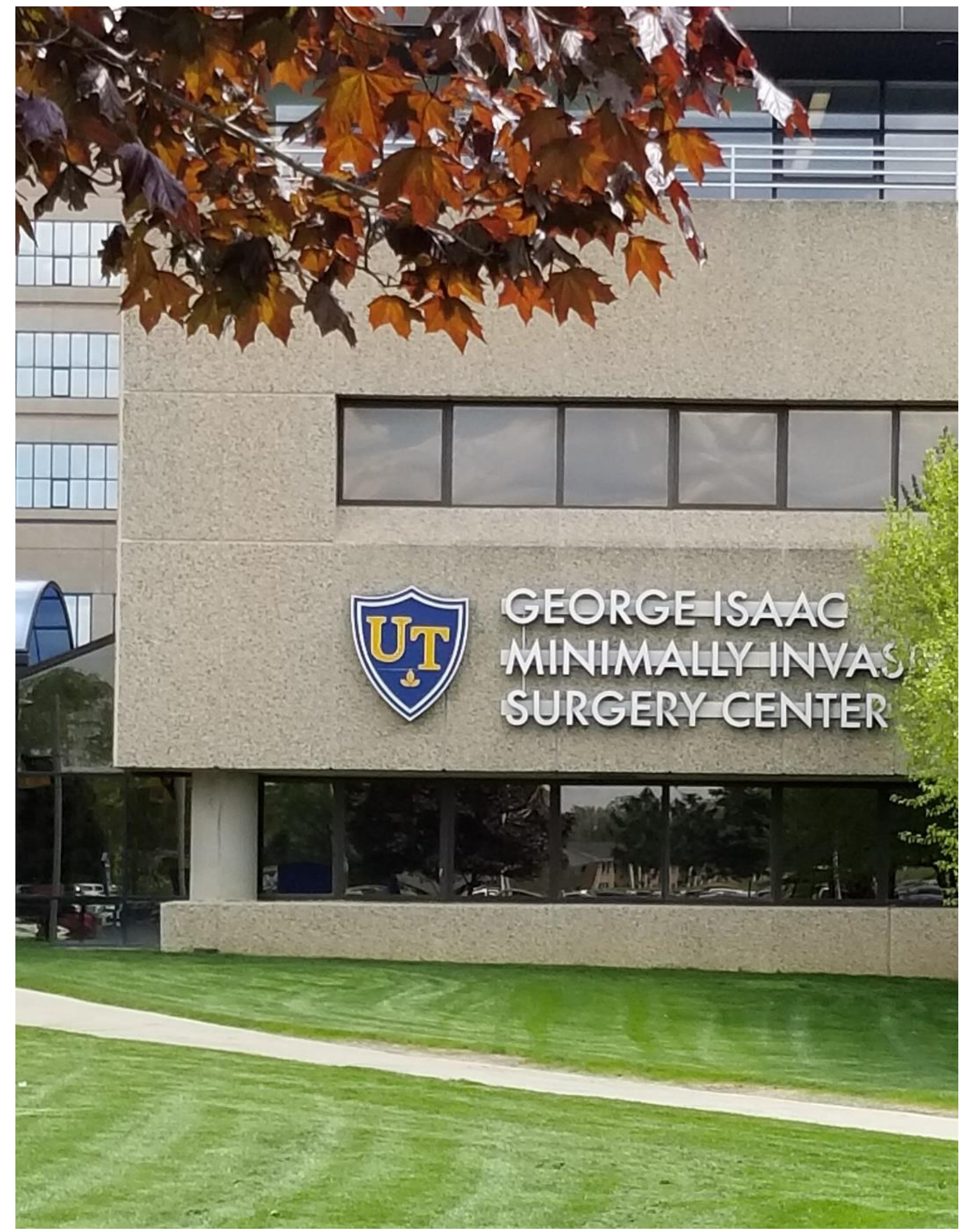




\title{
A case report of Clobazam toxicity related to Cannabidiol and Clobazam drug-drug interaction
}

\author{
Rowida Kheireldin ${ }^{a},{ }^{1}$ Naeem Mahfooz ${ }^{b}$
}

${ }^{a}$ Department of Pediatrics, University of Toledo Health Science Campus, Toledo, $\mathrm{OH}, \mathrm{USA}$, and ${ }^{b}$ Department of Pediatrics Neurology, University of Toledo, Toledo, OH, USA.

\begin{abstract}
Over the past few years, implementation of cannabis (CBD) as an anti-epileptic medication has been investigated in clinical research. CBD and Clobazam show remarkable antiepileptic efficacy in refractory epilepsies associated with Dravet and LennoxGastaut syndrome. There is a known drug-drug interaction between CBD and Clobazam however, there are no recommendations regarding dosing and monitoring of Clobazam while on CBD treatment. We present a 15-year-old female patient with a history of Dravet syndrome who presented to the emergency department with urinary retention and altered mental state four weeks after initiation of CBD treatment while on Clobazam.
\end{abstract}

| Epidiolex | Clobazam | Dravet syndrome | Lennox-Gastuat syndrome |

he interest in the anti-epileptic efficacy of cannabinoids (CBD) has significantly increased over the last 5 years. Trials have shown a remarkable reduction in seizure frequency in patient population of Dravet and Lennox-Gastaut syndromes using CBD. Clobazam is an important anti-epileptic medication with a special role in controlling epileptic drop attacks in these patients. CBD reacts with Clobazam leading to a significant increase in the levels of N-desmethylclobazam, the active metabolite of Clobazam. We present a case of a patient with Dravet syndrome who presented to the emergency department with urinary retention and altered mental state secondary to Clobazam toxicity related to CBD and Clobazam drug-drug interaction.

\section{Case report}

Patient information. Age: 15 years old. Gender: female. Ethnicity: caucasian. Related medical problems: Dravet syndrome, hypotonia, and global developmental delay.

Objective. Clobazam toxicity can occur secondary to CBD due to drug-drug interaction since CBD inhibits the activity of cytochrome P2C19 enzymes leading to a significant elevation of $\mathrm{N}$ desmethylclobazam levels with clinical signs of urinary retention followed by alteration in mentation. The purpose of this case report is to focus on Clobazam toxicity secondary to this interaction and to signify the importance of monitoring Clobazam and $\mathrm{N}$ desmethylclobazam levels in patients concomitantly using CBD and Clobazam. We suggest decreasing the dose of Clobazam to half of the maintenance dose upon initiating CBD treatment. We also recommend monitoring Clobazam levels every two weeks for the first few months to avoid side effects and toxicity.

Case. A 15-year-old female with a past medical history of Dravet syndrome, hypotonia, and global developmental delay presented to the pediatrics emergency department with altered mental status, urinary retention and bowel incontinence. Symptoms started after the patient started Epidiolex (CBD) $160 \mathrm{mg}$ daily four weeks before presentation with no additional dose changes. She was on multiple antiepileptic drugs including Clobazam $80 \mathrm{mg}$ daily, Ethosuximide $250 \mathrm{mg}$ daily, Topiramate $200 \mathrm{mg}$ daily and potassium bromide $2 \mathrm{mg}$ daily. Her seizure frequency dropped from daily to no further seizures since starting Epidiolex however, her mental status started deteriorating and the patient became lethargic and confused. Her baseline was independence in daily life activities as she lived at a home health institute. Her caregivers reported that she had progressively worsened and became dependent. Her first symptom was urinary retention, which lead to an initial emergency department presentation two weeks prior to this admission. Urinalysis was done and it came back normal, thus the patient was sent home. Symptoms continued and progressively worsened as she developed an unsteady gait, bowel and bladder incontinence along with confusion and increased sleepiness.

On admission, the patient was disoriented to time, place and person with Glasgow coma scale of 12 . She received normal saline bolus, and Foley catheter was placed. Brain CT scan result was normal. Her EEG showed moderate diffuse encephalopathy with background and intermittent slowing with generalized poly-spikes and waves. Clobazam was immediately discontinued due to the patient's altered mental status. Her other home medications were continued. Lab results revealed topiramate level of $20.2 \mathrm{mg} / \mathrm{mL}$ (usual range $5-20 \mathrm{mg} / \mathrm{mL}$ ), bromide at $138.6 \mathrm{mg} / \mathrm{dL}$ (usual range $75-150$ $\mathrm{mg} / \mathrm{dL}$ ) and hyperchloremia on basal metabolic panel.

Topiramate toxicity was excluded. Differential diagnosis of Acute Disseminated Encephalomyelitis and Acute Flaccid Myelitis were ruled out by normal MRI brain and spine. Clobazam and $\mathrm{N}$ desmethylclobazam levels were found elevated at $512 \mathrm{ng} / \mathrm{mL}$ (usual range $30-300 \mathrm{ng} / \mathrm{mL}$ ) and $15,020 \mathrm{ng} / \mathrm{mL}$ (usual range 300 to 3,000 $\mathrm{ng} / \mathrm{mL}$ ) respectively. There was no baseline Clobazam level obtained prior to starting Epidiolex. The patient significantly improved through the remainder of her hospital course with marked improvement in her mental state within four days. Her urinary retention resolved in 3 day after discontinuing Clobazam.

She was discharged home, off of Clobazam and was given a follow up appointment with neurology in 1 week. Upon following up as outpatient; her mental status was back to normal. Her Clobazam was not restarted, instead she was placed on Perampanel $2 \mathrm{mg} /$ day.

All authors contributed to this paper. ${ }^{1}$ To whom correspondence should be sent: Rowida.Kheireldin@UToledo.Edu

Authors declare no conflict of interest. Submitted: 10/16/2019, published: 11/15/2019.

Freely available online through the UTJMS open access option 
There was no repeat Clobazam or N-desmethylclobazam levels for follow up since Clobazam was discontinued and she continued to show improvement in her mental status. No genetic testing for our patient was obtained including genetic polymorphism for CYP2C19 expression.

\section{Discussion}

Multiple trials have shown that CBD can reduce the frequency of convulsive seizures in patients with Dravet syndrome, and may limit epileptic drop attacks in patients with Lennox-Gastaut syndrome (2). Clobazam is frequently co-administered with CBD, especially in patients with Dravet and Lennox-Gastaut syndromes since this combination has shown to cause a significant decrease in motor seizures $(2,3)$. Geffrey et al. reported in a case report that the combination of CBD and Clobazam can lead to more than 50\% decrease in seizures in patient population (1). In our case report, the patient had no seizures reported after she was started on CBD. A common adverse event reported with CBD is sedation, being more frequent in patients taking Clobazam concomitantly. The onset of sedation as a result of the interaction with Clobazam mostly occurs within the first two weeks of initiation of therapy and usually resolves after lowering the Clobazam dose $(2,5)$.

In our case, the patient had urinary retention as her first symptom which appeared within two weeks of initiating CBD and her mentation progressively worsened over four weeks after starting her CBD treatment. Patients with refractory epilepsy on CBD and Clobazam were reported to have elevated levels of Clobazam and $\mathrm{N}$-desmethylclobazam, its active metabolite (1). This interaction is secondary to the fact that both medications are metabolized by cytochrome P450 enzymes and glucuronyl transferases. CBD inhibits the activity of cytochrome P2C19 enzymes leading to a significant elevation of $\mathrm{N}$-desmethylclobazam levels (6). In the study by Geffrey et al. the combination of CBD and Clobazam resulted in elevation of the levels of Clobazam by $60-80 \%$, as well as an increase in the N-desmethylclobazam by $300-500 \%$ (1). There are no significant data regarding the relation between CBD dosing and the drug-drug interaction between CBD and Clobazam.

Devinsky et al. reported that in patients with Dravet syndrome, the concentrations of $\mathrm{N}$-desmethylclobazam increased regardless of the CBD dose given (7). However, Chang et al study demonstrated elevation of N-desmethylclobazam levels with increasing the dose of CBD (8). The aim of this case report is to focus on the recommendations to modify the Clobazam dose in patients on CBD. The

1. Geffrey AL, Pollack SF, Bruno PL, Thiele EA. (2015) Drug-drug interaction between Clobazam and Cannabidiol in Children with Refractory Epilepsy 56(8):1246-51.

2. Perucca E. (2017) Cannabinoids in the Treatment of Epilepsy: Hard Evidence at Last? J Epilepsy Res. 7(2): 61\{76.

3. Bruno P, Savage T, Skirvin L, Wolper E, Thiele E. (2017) Efficacy of Cannabidiol in Patients with Refractory Epilepsy Relative to Concomitant Use of Clobazam. American epilepsy society. Annual meeting abstracts (Abst. 3.181).

4. Klotz KA, Schulze-Bonhage A, Antonio-Arce VS, Jacobs J. (2018) Cannabidiol for Treatment of Childhood Epilepsy-A Cross-Sectional Survey. Front Neurol 7;9:731.

5. Iffland K, Grotenhermen F.(2017) An Update on Safety and Side Effects of Cannabidiol: A Review of Clinical Data and Relevant Animal Studies. Cannabis Cannabinoid Res. 1;2(1):139- 154. rationale behind these adjustments is to avoid side effects of sedation, urinary retention and altered mental status, such as reported in our patient. There was a plan to reduce her Clobazam dose at the time of starting her on CBD, however she was admitted to hospital prior to the next follow up.

We suggest decreasing the dose of Clobazam to half of the maintenance dose upon initiating CBD treatment. This prompts emphasis on the relevance of monitoring Clobazam and Ndesmethylclobazam levels in patients using the combination of CBD and Clobazam $(1,9)$. There were no follow up levels obtained for Clobazam and N-desmeythlclobazam in our patient. We recommend obtaining baseline Clobazam level before starting CBD and monitoring Clobazam levels every two weeks for a few months to avoid side effects and toxicity. Going forward, randomized controlled trials are necessary to look into the required adjustments of Clobazam dose when used concomitantly with CBD. Also, further studies are needed to determine the relevance of monitoring Clobazam and N-desmethylclobazam levels in patients using the combination of CBD and Clobazam.

\section{Conclusion}

CBD can cause a remarkable increase in the N-desmethylclobazam levels, the active metabolite of Clobazam, which can lead to toxicity with clinical sings of urinary retention followed by alteration in mentation. It is significantly important to monitor blood levels for Clobazam and N-desmethylclobazam before the start of CBD in patients on Clobazam. We suggest decreasing the dose of Clobazam to half of the maintenance dose upon initiating CBD treatment. We also recommend monitoring Clobazam levels every two weeks in the first few months to avoid side effects and toxicity. Additional well controlled studies are needed to establish the recommended doses and the management of the doses of other concomitant anti-epileptic medications with CBD use.

\section{Conflict of interest}

Authors declare no conflict of interest.

\section{Authors contributions}

RK wrote the manuscript, NM revised the manuscript. All authors have read and approved the final document.

6. Gaston TE, Szaflarski JP. (2018) Cannabis for the Treatment of Epilepsy: an Update. Curr Neurol Neurosci Rep. 8;18(11):73.

7. Devinsky O, Patel AD, Thiele EA, Wong MH, Appleton R, et al. (2018) GWPCARE1 Part A Study Group. Randomized, dose-ranging safety trial of Cannabidiol in Dravet syndrome. Neurology. 3;90(14):e1204-e1211.

8. Chang B. (2018) Cannabidiol and Serum Antiepileptic Drug Levels: The ABCs of CBD With AEDs. Epilepsy Curr. 18(1): $33\{34$.

9. Gaston, Bebin E, Szaflarski J. (2016) Importance of Monitoring Clobazam and NDesmethylclobazam Levels in Treatment with Cannabidiol (CBD) for Epilepsy. Neurology, Apr 2016, 86 (16 Supplement) S14.001. 